\title{
Mesoscopic lattice Boltzmann model for radiative heat transfer in graded-index media
}

\author{
Xiaochuan Liu, ${ }^{*} \mathrm{Si} \mathrm{Wu},{ }^{*}$ Keyong Zhu $\odot,{ }^{\dagger}$ Yuepei Cai, and Yong Huang $\oplus^{\ddagger}$ \\ School of Aeronautic Science and Engineering, Beihang University, Beijing 100191, China
}

(Received 6 October 2021; accepted 8 February 2022; published 16 February 2022)

\begin{abstract}
Convection, conduction, and thermal radiation are the three mechanisms of heat transfer in nature. The lattice Boltzmann model (LBM) has already achieved great success in dealing with convection and conduction problems. However, the mature LBM for radiative heat transfer (RHT) is still relatively lacking. Here we propose a mesoscopic LBM for RHT in graded-index media, which enables a simple and efficient solution of both transient and steady-state RHT in graded-index media by conducting collision and streaming processes. Via the Chapman-Enskog analysis, the radiative transfer equation of graded-index media is rigorously derived from the proposed LBM. The present LBM is a universal model for RHT in media with arbitrary refractive index distribution, which can naturally handle RHT in homogeneous media with constant refractive index. This model is expected to provide a simple and efficient mesoscopic tool for RHT in complex media and pave the way for establishing a unified framework of LBM for convection, conduction, and thermal radiation heat transfer.
\end{abstract}

DOI: 10.1103/PhysRevResearch.4.013125

\section{INTRODUCTION}

Since its introduction three decades ago [1,2], the lattice Boltzmann model (LBM) has developed into a recognized and popular approach for computational fluid dynamics [3-5], with various applications including magnetohydrodynamics [6], turbulent flow [7], multiphase flow [8], micro- and nanoscale flow [9], thermal flow [10,11], relativistic hydrodynamics [12] and so on [13-17]. In conjunction with the great success in hydrodynamics, the LBM has also been successfully extended to macroscopic thermal conduction [18-20], mesoscopic phonon transport [21-23]. Due to its kinetic origin, the LBM is a mesoscopic approach that possesses the prominent features of simple formulation and implementation as well as high parallel efficiency. Its attractive features drove the LBM to be further introduced into many other fields, such as convection-diffusion [24], reaction-diffusion [25], wave propagation [26], and quantum mechanics [27].

Inspired by wide applications of the LBM in convection and conduction, numerous researchers have promoted the application of the LBM to radiative heat transfer (RHT) over the past decade [28-38] and have been devoted to establishing a unified framework of the LBM for convection, conduction, and thermal radiation heat transfer. However, all above-mentioned LBM for RHT are intended for simple homogeneous media with constant refractive index, and they are not applicable to complex media with variable refractive

\footnotetext{
${ }^{*}$ These authors contributed equally to this work.

†hukeyong@buaa.edu.cn

†huangy@buaa.edu.cn
}

Published by the American Physical Society under the terms of the Creative Commons Attribution 4.0 International license. Further distribution of this work must maintain attribution to the author(s) and the published article's title, journal citation, and DOI. index. Essentially, in real physical media, variable refractive index (i.e., graded index) is universal, while the constant refractive index is only special simplification [39,40]. RHT in graded-index media widely appears in many scientific and engineering fields, such as combustion [41], atmospheric radiative transfer [42], optical measurement [39], as well as thermo-optical systems [43]. In the past two decades, it has attracted a wide range of attention and some numerical methods have been developed to investigate RHT in graded-index media [44-53], but these methods are relatively difficult to deal with conjugated convection, conduction, and thermal radiation heat transfer in a unified manner. Overall, it is of great significance to develop a LBM for RHT in graded-index media, not only to achieve a simple and efficient solution to the problem, but also to pave the way for establishing a unified framework of the LBM for convection, conduction, and thermal radiation heat transfer.

Due to its complexity, the LBM has rarely been applied to RHT in graded-index media. Zhang et al. [54] developed a pioneering LBM for one-dimensional transient RHT in graded-index media. They constructed the lattice Boltzmann equation (LBE) by discretizing the radiative transfer equation (RTE) in time and space. The mesoscopic equilibrium distribution function in the LBE and the rigorous link between the LBE and RTE have not been established. So far, a mature mesoscopic LBM for RHT in graded-index media is still relatively lacking and thus urgently required.

In this work, we propose a mesoscopic LBM for RHT in graded-index media. Our discussion and implementation are limited to the gray RTE. We construct the LBE for the RTE and the equilibrium distribution function for the radiative intensity, respectively. The RTE in graded-index media is rigorously derived from the proposed LBM via the Chapman-Enskog (CE) analysis. The present LBM shows a clear physical picture at the mesoscopic level, and we demonstrate that it enables a simple and efficient solution of both transient and steady-state RHT in graded-index media. Also, 
we note that the present LBM can be naturally degenerated to handle RHT in homogeneous media with constant refractive index.

\section{PHYSICAL MODEL AND MATHEMATICAL FORMULATIONS}

\section{A. RTE in graded-index media}

The RTE in this work is restricted to the common gray (frequency-independent approximation) form, and the gray RTE for RHT in absorbing, emitting, and scattering media with graded index can be expressed as [48]

$$
\begin{aligned}
\frac{n}{c_{l}} \partial_{t} I(\mathbf{r}, \boldsymbol{\Omega}, t)+\boldsymbol{\Omega} \cdot \nabla I(\mathbf{r}, \boldsymbol{\Omega}, t) \\
\quad+\frac{1}{2 n^{2} \sin \theta} \partial_{\theta}\left[I(\mathbf{r}, \boldsymbol{\Omega}, t)(\xi \boldsymbol{\Omega}-\mathbf{k}) \cdot \nabla n^{2}\right] \\
\quad+\frac{1}{2 n^{2} \sin \theta} \partial_{\varphi}\left[I(\mathbf{r}, \boldsymbol{\Omega}, t)\left(\mathbf{s}_{1} \cdot \nabla n^{2}\right)\right]+k_{e} I(\mathbf{r}, \boldsymbol{\Omega}, t) \\
=n^{2} k_{a} I_{b}(\mathbf{r}, t)+\frac{k_{s}}{4 \pi} \int_{4 \pi} I\left(\mathbf{r}, \boldsymbol{\Omega}^{\prime}, t\right) \Phi\left(\boldsymbol{\Omega}^{\prime}, \boldsymbol{\Omega}\right) d \mathbf{\Omega}^{\prime}
\end{aligned}
$$

where $c_{l}$ is the speed of light in vacuum; $n$ is the refractive index of media, which is a function of spatial position $\mathbf{r} ; \boldsymbol{\Omega}=\mu \mathbf{i}+\eta \mathbf{j}+\xi \mathbf{k}=\cos \varphi \sin \theta \mathbf{i}+\sin \varphi \sin \theta \mathbf{j}+\cos \theta \mathbf{k}$ is the radiation direction with $\mu, \eta$, and $\xi$ being the direction cosine in Cartesian coordinate, and $\theta$ and $\varphi$ being the zenith and azimuthal angles, respectively; $t$ is the time; $I$ is the radiative intensity; $I_{b}$ is the blackbody radiative intensity; $k_{e}, k_{a}$, and $k_{s}$ are the extinction, absorption, and scattering coefficients, respectively, and $k_{e}=k_{a}+k_{s} ; \Phi\left(\boldsymbol{\Omega}^{\prime}, \boldsymbol{\Omega}\right)$ is the scattering phase function from the incoming direction $\boldsymbol{\Omega}^{\prime}$ to the outgoing direction $\boldsymbol{\Omega} ; \mathbf{s}_{1}$ is a vector defined as $\mathbf{s}_{1}=-\sin \varphi \mathbf{i}+\cos \varphi \mathbf{j}$.

For the boundary surfaces with diffuse and specular reflection and collimated irradiation, the boundary condition for RTE can be written as

$$
\begin{aligned}
I\left(\mathbf{r}_{w}, \boldsymbol{\Omega}, t\right)= & \left(f_{d} \varepsilon_{d}+f_{s} \varepsilon_{s}\right) n_{w}^{2} I_{b, w}\left(\mathbf{r}, t^{*}\right) \\
& +\frac{f_{d} \rho_{d}}{\pi} \int_{\mathbf{n}_{w} \cdot \boldsymbol{\Omega}^{\prime}<\mathbf{0}} I\left(\mathbf{r}_{w}, \boldsymbol{\Omega}^{\prime}, t\right)\left|\mathbf{n}_{w} \cdot \boldsymbol{\Omega}^{\prime}\right| \mathrm{d} \boldsymbol{\Omega}^{\prime} \\
& +f_{s} \rho_{s} I\left(\mathbf{r}_{w}, \boldsymbol{\Omega}^{\prime \prime}, t\right)+\left[f_{d}\left(1-\rho_{d}\right)\right. \\
& \left.+f_{s}\left(1-\rho_{s}\right)\right] I^{\mathrm{ext}}\left(\mathbf{r}_{w}, \boldsymbol{\Omega}_{c}, t\right)
\end{aligned}
$$

where the subscript " $w$ " denotes the boundary; $\varepsilon_{d}$ and $\varepsilon_{s}$ are the diffuse and specular emissivity, respectively; $\rho_{d}$ and $\rho_{s}$ are the diffuse and specular reflectivity, respectively; $f_{d}$ and $f_{s}$ are the fractions of the diffuse and specular reflection, respectively, and $f_{d}+f_{s}=1 ; \Omega^{\prime}, \Omega^{\prime \prime}$, and $\Omega_{c}$ are the incident angle of diffuse reflection, specular reflection, and collimated irradiation, respectively; $n_{w}$ is the unit inner direction of the boundary surface; $I^{\text {ext }}$ denotes external irradiation intensity.

\section{B. LBM for RHT in graded-index media}

Next, a LBM for RHT in graded-index media will be proposed. Since the refractive index of media is a function of spatial position, our key idea is to introduce the local radiative transfer direction $\mathbf{u}=\mathbf{\Omega} / n$ and act the gradient operator onto it. Introducing the dimensionless time $t^{*}=c_{l} t / L$, the temporally dimensionless $\mathrm{RTE}$ can be obtained as

$$
\partial_{t^{*}} I\left(\mathbf{r}, \boldsymbol{\Omega}, t^{*}\right)+\nabla \cdot\left[L \mathbf{u} I\left(\mathbf{r}, \boldsymbol{\Omega}, t^{*}\right)\right]=F\left(\mathbf{r}, \boldsymbol{\Omega}, t^{*}\right),
$$

with

$$
F\left(\mathbf{r}, \boldsymbol{\Omega}, t^{*}\right)=L\left[S-k_{e} I\left(\mathbf{r}, \boldsymbol{\Omega}, t^{*}\right)\right] / n+I\left(\mathbf{r}, \boldsymbol{\Omega}, t^{*}\right) \nabla \cdot(L \mathbf{u}),
$$

and

$$
\begin{aligned}
S= & n^{2} k_{a} I_{b}\left(\mathbf{r}, t^{*}\right)+\frac{k_{s}}{4 \pi} \int_{4 \pi} I\left(\mathbf{r}, \boldsymbol{\Omega}^{\prime}, t^{*}\right) \Phi\left(\boldsymbol{\Omega}^{\prime}, \boldsymbol{\Omega}\right) \mathrm{d} \boldsymbol{\Omega}^{\prime} \\
& -\frac{1}{2 n^{2} \sin \theta} \partial_{\theta}\left[I\left(\mathbf{r}, \boldsymbol{\Omega}, t^{*}\right)(\xi \boldsymbol{\Omega}-\mathbf{k}) \cdot \nabla n^{2}\right] \\
& -\frac{1}{2 n^{2} \sin \theta} \partial_{\varphi}\left[I\left(\mathbf{r}, \boldsymbol{\Omega}, t^{*}\right)\left(\mathbf{s}_{1} \cdot \nabla n^{2}\right)\right],
\end{aligned}
$$

where $L$ is the reference length of media. Note that $I\left(\mathbf{r}, \boldsymbol{\Omega}, t^{*}\right) \nabla \cdot(L \mathbf{u})$ in source term $F\left(\mathbf{r}, \boldsymbol{\Omega}, t^{*}\right)$ is complemented by acting the gradient operator on the local radiative transfer direction.

Here we give the corresponding LBE for the temporally dimensionless RTE, Eq. (3), as

$$
\begin{gathered}
f_{i}\left(\mathbf{r}+\mathbf{c}_{i} \Delta t^{*}, \boldsymbol{\Omega}, t^{*}+\Delta t^{*}\right)-f_{i}\left(\mathbf{r}, \boldsymbol{\Omega}, t^{*}\right) \\
=-\frac{1}{\tau}\left[f_{i}\left(\mathbf{r}, \boldsymbol{\Omega}, t^{*}\right)-f_{i}^{\mathrm{eq}}\left(\mathbf{r}, \boldsymbol{\Omega}, t^{*}\right)\right] \\
+\Delta t^{*}\left[A_{i}\left(\mathbf{r}, \boldsymbol{\Omega}, t^{*}\right)+F_{i}\left(\mathbf{r}, \boldsymbol{\Omega}, t^{*}\right)\right. \\
\left.+\frac{\Delta t^{*}}{2} \partial_{t^{*}} F_{i}\left(\mathbf{r}, \boldsymbol{\Omega}, t^{*}\right)\right],
\end{gathered}
$$

where $\tau$ denotes the dimensionless relaxation time; $\Delta t^{*}$ denotes the dimensionless time step; $\mathbf{c}_{i}$ denotes the discrete lattice velocity; $f_{i}\left(\mathbf{r}, \boldsymbol{\Omega}, t^{*}\right)$ and $f_{i}^{\mathrm{eq}}\left(\mathbf{r}, \boldsymbol{\Omega}, t^{*}\right)$ denote the distribution function and equilibrium distribution function in discrete velocity space, respectively; $F_{i}\left(\mathbf{r}, \boldsymbol{\Omega}, t^{*}\right)$ denotes the source term distribution function; and $A_{i}\left(\mathbf{r}, \boldsymbol{\Omega}, t^{*}\right)$ denotes the auxiliary source term distribution function. Noted that $\partial_{t^{*}} F_{i}\left(\mathbf{r}, \boldsymbol{\Omega}, t^{*}\right)=\left[F_{i}\left(\mathbf{r}, \boldsymbol{\Omega}, t^{*}\right)-F_{i}\left(\mathbf{r}, \boldsymbol{\Omega}, t^{*}-\Delta t^{*}\right)\right] / \Delta t^{*}$.

The equilibrium distribution function in discrete velocity space follows as $[37,38]$

$$
\begin{aligned}
f_{i}^{\mathrm{eq}}\left(\mathbf{r}, \boldsymbol{\Omega}, t^{*}\right)= & \varpi_{i} I\left(\mathbf{r}, \boldsymbol{\Omega}, t^{*}\right)\left[1+\frac{\mathbf{c}_{i} \cdot(L \mathbf{u})}{c_{s}^{2}}\right. \\
& \left.+\frac{\left(L^{2} \mathbf{u u}-c_{s}^{2} \mathbf{E}\right):\left(\mathbf{c}_{i} \mathbf{c}_{i}-c_{s}^{2} \mathbf{E}\right)}{2 c_{s}^{4}}\right],
\end{aligned}
$$

where $\varpi_{i}$ denotes the weight coefficient corresponding to discrete lattice velocity $\mathbf{c}_{i} ; c_{s}$ denotes the lattice sound speed; and $\mathbf{E}$ denotes the unite tensor.

The distribution functions of source term and auxiliary source term are, respectively, taken as

$$
\begin{aligned}
F_{i}\left(\mathbf{r}, \boldsymbol{\Omega}, t^{*}\right)= & \varpi_{i} F\left(\mathbf{r}, \boldsymbol{\Omega}, t^{*}\right), \\
A_{i}\left(\mathbf{r}, \boldsymbol{\Omega}, t^{*}\right)= & \lambda \varpi_{i} \mathbf{c}_{i} \cdot(L \mathbf{u})\left[\mathbf{F}\left(\mathbf{r}, \boldsymbol{\Omega}, t^{*}\right)\right. \\
& \left.+I\left(\mathbf{r}, \boldsymbol{\Omega}, t^{*}\right) \nabla \cdot(L \mathbf{u})\right] / c_{s}^{2},
\end{aligned}
$$

where $\lambda=1-1 /(2 \tau)$. The construction of the auxiliary source term distribution function is important in the present 
(a)

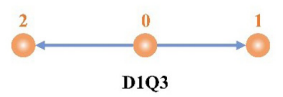

(b)

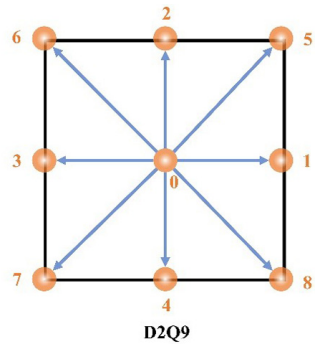

FIG. 1. Schematic of the D1Q3 and D2Q9 lattice structures.

LBM and used to modify the effect of gradient operator on the local radiative transfer direction.

The $\mathrm{D} d \mathrm{Q} q$ lattice modes are employed for different dimensional configurations. In this work, the D1Q3 and D2Q9 models [15] are adopted, the structure of which is shown in Fig. 1. The parameters of D1Q3 model are given by

$$
\begin{aligned}
& c_{i}= \begin{cases}0, & i=0 \\
\pm c, & i=1,2,\end{cases} \\
& c_{s}=\frac{c}{\sqrt{3}}, \quad \varpi_{i}= \begin{cases}2 / 3, & i=0 \\
1 / 6, & i=1,2 .\end{cases}
\end{aligned}
$$

The parameters of the D2Q9 model are given by

$$
\begin{gathered}
\mathbf{c}_{i}= \begin{cases}(0,0) c, & i=0 \\
\left(\cos (i-1) \frac{\pi}{2}, \sin (i-1) \frac{\pi}{2}\right) c, & i=1-4, \\
\sqrt{2}\left(\cos (2 i-1) \frac{\pi}{4}, \sin (2 i-1) \frac{\pi}{4}\right) c, & i=5-8\end{cases} \\
c_{s}=\frac{c}{\sqrt{3}}, \quad \varpi_{i}= \begin{cases}4 / 9, & i=0 \\
1 / 9, & i=1-4 . \\
1 / 36, & i=5-8\end{cases}
\end{gathered}
$$

Here $c$ is the lattice velocity defined as $c=\Delta x / \Delta t^{*}$, and $\Delta x^{*}$ denotes the dimensionless lattice size. For numerical stability, the dimensionless time step and lattice size should meet the Courant-Friedrichs-Lewy (CFL) condition [55], i.e., CFL coefficient $\mathrm{CFL} \leqslant 1\left(\mathrm{CFL}=\Delta t^{*} / \Delta x\right)$.

\section{Recovery of RTE from LBE}

For the mesoscopic LBM, the key is to link the RTE with the LBE. It depends on expressions of the equilibrium distribution function, source term, and auxiliary source term distribution functions. Here a commonly used method, i.e., the CE analysis, is employed to recover the RTE, Eq. (3), for graded-index media from the LBE, Eq. (4).

The distribution function, equilibrium distribution function, source term, and auxiliary source term distribution functions satisfy the following constrains:

$$
\begin{aligned}
\sum_{i} f_{i}\left(\mathbf{r}, \boldsymbol{\Omega}, t^{*}\right) & =\sum_{i} f_{i}^{\mathrm{eq}}\left(\mathbf{r}, \boldsymbol{\Omega}, t^{*}\right)=I\left(\mathbf{r}, \boldsymbol{\Omega}, t^{*}\right), \\
\sum_{i} \mathbf{c}_{i} f_{i}^{\mathrm{eq}}\left(\mathbf{r}, \boldsymbol{\Omega}, t^{*}\right) & =I\left(\mathbf{r}, \boldsymbol{\Omega}, t^{*}\right) L \mathbf{u}, \\
\sum_{i} \mathbf{c}_{i} \mathbf{c}_{i} f_{i}^{\mathrm{eq}}\left(\mathbf{r}, \boldsymbol{\Omega}, t^{*}\right) & =I\left(\mathbf{r}, \boldsymbol{\Omega}, t^{*}\right) L^{2} \mathbf{u u}, \\
\sum_{i} F_{i}\left(\mathbf{r}, \boldsymbol{\Omega}, t^{*}\right) & =F\left(\mathbf{r}, \boldsymbol{\Omega}, t^{*}\right), \quad \sum_{i} \mathbf{c}_{i} F_{i}\left(\mathbf{r}, \boldsymbol{\Omega}, t^{*}\right)=0,
\end{aligned}
$$

$$
\begin{aligned}
\sum_{i} A_{i}\left(\mathbf{r}, \boldsymbol{\Omega}, t^{*}\right) & =0, \\
\sum_{i} \mathbf{c}_{i} A_{i}\left(\mathbf{r}, \boldsymbol{\Omega}, t^{*}\right) & =\lambda \operatorname{Lu}[ \\
& F\left(\mathbf{r}, \boldsymbol{\Omega}, t^{*}\right) \\
& \left.+I\left(\mathbf{r}, \boldsymbol{\Omega}, t^{*}\right) \nabla \cdot(L \mathbf{u})\right] .
\end{aligned}
$$

For simplicity, the notations $f_{i}, f_{i}^{\mathrm{eq}}, F_{i}$, and $A_{i}$ are adopted in place of $f_{i}\left(\mathbf{r}, \boldsymbol{\Omega}, t^{*}\right), f_{i}^{\mathrm{eq}}\left(\mathbf{r}, \boldsymbol{\Omega}, t^{*}\right), F_{i}\left(\mathbf{r}, \boldsymbol{\Omega}, t^{*}\right)$, and $A_{i}\left(\mathbf{r}, \boldsymbol{\Omega}, t^{*}\right)$, respectively. Firstly, the CE expansion of time and space is applied:

$$
\begin{aligned}
f_{i} & =f_{i}^{\mathrm{eq}}+\varepsilon f_{i}^{(1)}+\varepsilon^{2} f_{i}^{(2)}+\mathrm{O}\left(\varepsilon^{3}\right), \\
F_{i} & =\varepsilon F_{i}^{(1)}+\varepsilon^{2} F_{i}^{(2)}+\mathrm{O}\left(\varepsilon^{3}\right), \\
A_{i} & =\varepsilon A_{i}^{(1)}+\varepsilon^{2} A_{i}^{(2)}+\mathrm{O}\left(\varepsilon^{3}\right), \\
F & =\varepsilon F^{(1)}+\varepsilon^{2} F^{(2)}+\mathrm{O}\left(\varepsilon^{3}\right), \\
\partial_{t^{*}} & =\varepsilon \partial_{t_{1}^{*}}+\varepsilon^{2} \partial_{t_{2}^{*}}+\mathrm{O}\left(\varepsilon^{3}\right), \\
\nabla & =\varepsilon \nabla_{1}+\mathrm{O}\left(\varepsilon^{2}\right),
\end{aligned}
$$

where the small expansion parameter $\varepsilon$ satisfies $\varepsilon \ll 1$.

The following relations can be obtained from Eqs. (9) and (10):

$$
\begin{aligned}
\sum_{i} f_{i}^{(m)} & =0, \quad \sum_{i} F_{i}^{(m)}=F^{(m)}, \quad \sum_{i} \mathbf{c}_{i} F_{i}^{(m)}=0 \\
\sum_{i} A_{i}^{(m)} & =0 \\
\sum_{i} \mathbf{c}_{i} A_{i}^{(1)} & =\lambda L \mathbf{u}\left[F^{(1)}+I\left(\mathbf{r}, \boldsymbol{\Omega}, t^{*}\right) \nabla_{1} \cdot(L \mathbf{u})\right],
\end{aligned}
$$

where $m=1,2$.

By applying the Taylor expansion to the evolution equation of the LBM, we have

$$
\begin{aligned}
D_{i} f_{i}+\frac{\Delta t^{*}}{2} D_{i}^{2} f_{i}+\cdots= & -\frac{1}{\tau \Delta t^{*}}\left(f_{i}-f_{i}^{\mathrm{eq}}\right)+F_{i}+A_{i} \\
& +\frac{\Delta t^{*}}{2} \partial_{t^{*}} F_{i},
\end{aligned}
$$

where $D_{i}=\partial_{t^{*}}+\mathbf{c}_{i} \cdot \nabla=\varepsilon D_{1 i}+\varepsilon^{2} \partial_{t_{2}^{*}} \quad$ and $\quad D_{1 i}=\partial_{t_{1}^{*}}+$ $\mathbf{c}_{i} \cdot \nabla_{1}$.

Substituting Eq. (10) into Eq. (12) and equating the coefficients of the same order of $\varepsilon$ and $\varepsilon^{2}$ leads to the following sequence of equations:

$$
\begin{aligned}
& \varepsilon: D_{1 i} f_{i}^{\mathrm{eq}}=-\frac{1}{\tau \Delta t^{*}} f_{i}^{(1)}+F_{i}^{(1)}+A_{i}^{(1)} \\
& \varepsilon^{2}: \partial_{t_{2}^{*}} f_{i}^{\mathrm{eq}}+D_{1 i} f_{i}^{(1)}+\frac{\Delta t^{*}}{2} D_{1 i}^{2} f_{i}^{\mathrm{eq}} \\
& \quad=-\frac{1}{\tau \Delta t^{*}} f_{i}^{(2)}+F_{i}^{(2)}+A_{i}^{(2)}+\frac{\Delta t^{*}}{2} \partial_{t_{1}^{*}} F_{i}^{(1)} .
\end{aligned}
$$

Applying Eqs. (13a) to (13b), and summing Eqs. (13a) and (13b) over the subscript $i$, and with the help of moment conditions in Eqs. (9) and (11), we obtain

$$
\begin{aligned}
& \partial_{t_{1}^{*}} I\left(\mathbf{r}, \boldsymbol{\Omega}, t^{*}\right)+\nabla_{1} \cdot\left(L \mathbf{u} I\left(\mathbf{r}, \boldsymbol{\Omega}, t^{*}\right)\right)=F^{(1)}, \\
& \partial_{t_{2}^{*}} I\left(\mathbf{r}, \boldsymbol{\Omega}, t^{*}\right)+\left(1-\frac{1}{2 \tau}\right) \nabla_{1}
\end{aligned}
$$




$$
\begin{aligned}
& \cdot \sum_{i} \mathbf{c}_{i} f_{i}^{(1)}+\left(1-\frac{1}{2 \tau}\right) \frac{\Delta t^{*}}{2} \nabla_{1} \\
& \cdot\left[L \mathbf{u}\left(F^{(1)}+I\left(\mathbf{r}, \boldsymbol{\Omega}, t^{*}\right) \nabla_{1} \cdot(L \mathbf{u})\right)\right]=F^{(2)} .
\end{aligned}
$$

Applying the expression of $f_{i}^{(1)}$ in Eq. (13a), and with the help of Eq. (14a), Eq. (14b) can be eventually expressed as

$$
\partial_{t_{2}^{*}} I=F^{(2)} \text {. }
$$

Taking $\varepsilon \times$ Eq. (14a) $+\varepsilon^{2} \times$ Eq. (15), we have

$$
\partial_{t^{*}} I\left(\mathbf{r}, \boldsymbol{\Omega}, t^{*}\right)+\nabla \cdot\left[L \mathbf{u} I\left(\mathbf{r}, \boldsymbol{\Omega}, t^{*}\right)\right]=F\left(\mathbf{r}, \boldsymbol{\Omega}, t^{*}\right) .
$$

Ultimately, the RTE for graded-index media is rigorously recovered from the $\mathrm{LBE}$ through the $\mathrm{CE}$ analysis.

\section{Boundary treatment}

The radiative intensity at the boundary of the upstream direction can be given in Eq. (2). For the downstream direction, the radiative intensity at the boundary can be obtained as

$$
I\left(\mathbf{r}_{w}, \boldsymbol{\Omega}, t^{*}\right) / n_{w}^{2}=2 I\left(\mathbf{r}_{a}, \boldsymbol{\Omega}, t^{*}\right) / n_{a}^{2}-I\left(\mathbf{r}_{n a}, \boldsymbol{\Omega}, t^{*}\right) / n_{n a}^{2},
$$

where $\mathbf{r}_{w}$ denotes the boundary node, $\mathbf{r}_{a}$ denotes the node neighboring the boundary node, and $\mathbf{r}_{n a}$ denotes the nextneighboring node of $\mathbf{r}_{a}$, which are shown in Fig. $2 ; n_{w}$, $n_{a}$, and $n_{n a}$ denote the refractive index of the corresponding nodes.

The distribution function at the boundary can be obtained by the nonequilibrium extrapolation scheme [56]:

$$
\begin{aligned}
& f_{i}\left(\mathbf{r}_{w}, \boldsymbol{\Omega}, t^{*}\right) \\
& \quad=f_{i}^{\mathrm{eq}}\left(\mathbf{r}_{w}, \boldsymbol{\Omega}, t^{*}\right)+\left[f_{i}\left(\mathbf{r}_{a}, \boldsymbol{\Omega}, t^{*}\right)-f_{i}^{\mathrm{eq}}\left(\mathbf{r}_{a}, \boldsymbol{\Omega}, t^{*}\right)\right] .
\end{aligned}
$$

Until now, we have given the mesoscopic LBM for RHT in graded-index media. Obviously, it is naturally applicable to RHT in homogeneous media with constant refractive index. Thus, the present LBM is a universal model for RHT in media with arbitrary refractive index.

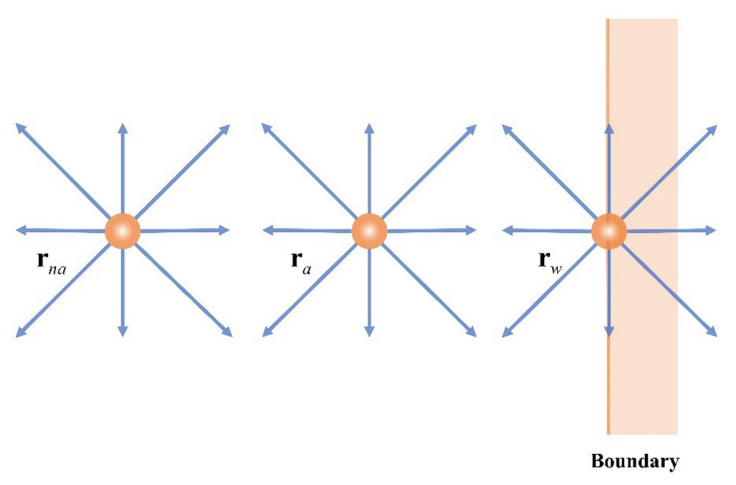

FIG. 2. Schematic of the boundary treatment with $\mathbf{r}_{w}, \mathbf{r}_{a}$, and $\mathbf{r}_{n a}$ representing the boundary lattice node, the adjacent lattice node, and the next adjacent lattice node, respectively.

\section{RESULTS AND DISCUSSION}

To test the applicability of present mesoscopic LBM for RHT in graded-index media, one-dimensional and twodimensional transient and steady-state cases are studied in this section. To decouple the space-angle dependency, the spatial domain and angular domain are divided into some lattices and discrete directions, respectively. The angular domain discretization scheme adopts the popular piecewise constant approximation scheme, and accordingly the derivative terms of refractive index to zenith and azimuth angles are processed by the classical finite-difference scheme [48]. After the lattice and discrete direction independence verifications, 200 uniform lattices and 80 zenith discrete directions are adopted for the one-dimensional cases, and $20 \times 20$ uniform lattices and $20 \times 40$ discrete directions are adopted for the two-dimensional cases. In this work, the dimensionless relaxation time is taken as $\tau=1.0$, and CFL is taken as 0.2. The LBM is a transient evolutionary algorithm, and the following time convergence criterion is executed for steady-state problems:

$$
\frac{\sum_{j}\left|G\left(\mathbf{r}_{j}, t^{*}\right)-G\left(\mathbf{r}_{j}, t^{*}-100 \Delta t^{*}\right)\right|}{\sum_{j} G\left(\mathbf{r}_{j}, t^{*}\right)}<10^{-6},
$$

where $G$ is the incident radiation. The solution steps of the present LBM for RHT can be seen in Ref. [36].

\section{A. One-dimensional cases}

The first case we consider is the transient RHT in an infinite slab with diffuse boundary radiation. The slab is absorbing, isotropic scattering, and nonemitting bounded by two black surfaces. The thickness of the slab is $L=1 \mathrm{~m}$, and the optical thickness is $\tau_{L}=\kappa_{e} L=1.0$. The single scattering albedo is $\omega=\kappa_{s} / \kappa_{e}=0.5$. The left boundary provides a diffuse intensity $I\left(0, \xi, t^{*}\right)=H\left(t^{*}\right) I_{0}$, where $H\left(t^{*}\right)$ is the Heaviside step function. The refractive index distribution is $n=1+2 z$. Figures 3(a) and 3(b) show time evolutionary processes of dimensionless incident radiation $G^{*}=2 \pi \int_{-1}^{1} I \mathrm{~d} \xi / \pi I_{0}$ and radiative heat flux $q^{*}=2 \pi \int_{-1}^{1} I|\xi| \mathrm{d} \xi / \pi I_{0}$ in the slab. The results of the present LBM exhibit good agreement with those of the discontinuous finite-element method (DFEM) [50]. Theoretically, the wavefront of the pulse irradiation will arrive at position $z$ at $t^{*}=\int_{0}^{z} n / \xi d z$ for different zenith directions $\xi$. We can see the wavefront with different $\xi$ for $t^{*}=0.4$, 1.0, 2.0, and steady state in Figs. 3(c)-3(f), which features a nonlinear relationship between $z$ and $\xi$, instead of the linear relationship for constant refractive index. Especially, $t^{*}=z+z^{2}$ for $\xi=1$, and the wavefront arrives at positions $z=0.306,0.618$, and 1.0, respectively, when $t^{*}=0.4,1.0$, and 2.0, consistent with the LBM results. To demonstrate the universality of present LBM for RHT, we further consider the slab with a constant refractive index $n=1$, and other parameters remain unchanged. Time evolutionary processes of incident radiation and radiative heat flux in the homogeneous slab are shown in Fig. 4. The LBM results also exhibit good agreement with the DFEM results [57], which indicates that the present LBM can be naturally degenerated to handle RHT in homogeneous media with constant refractive index by taking the global radiative transfer direction and is 

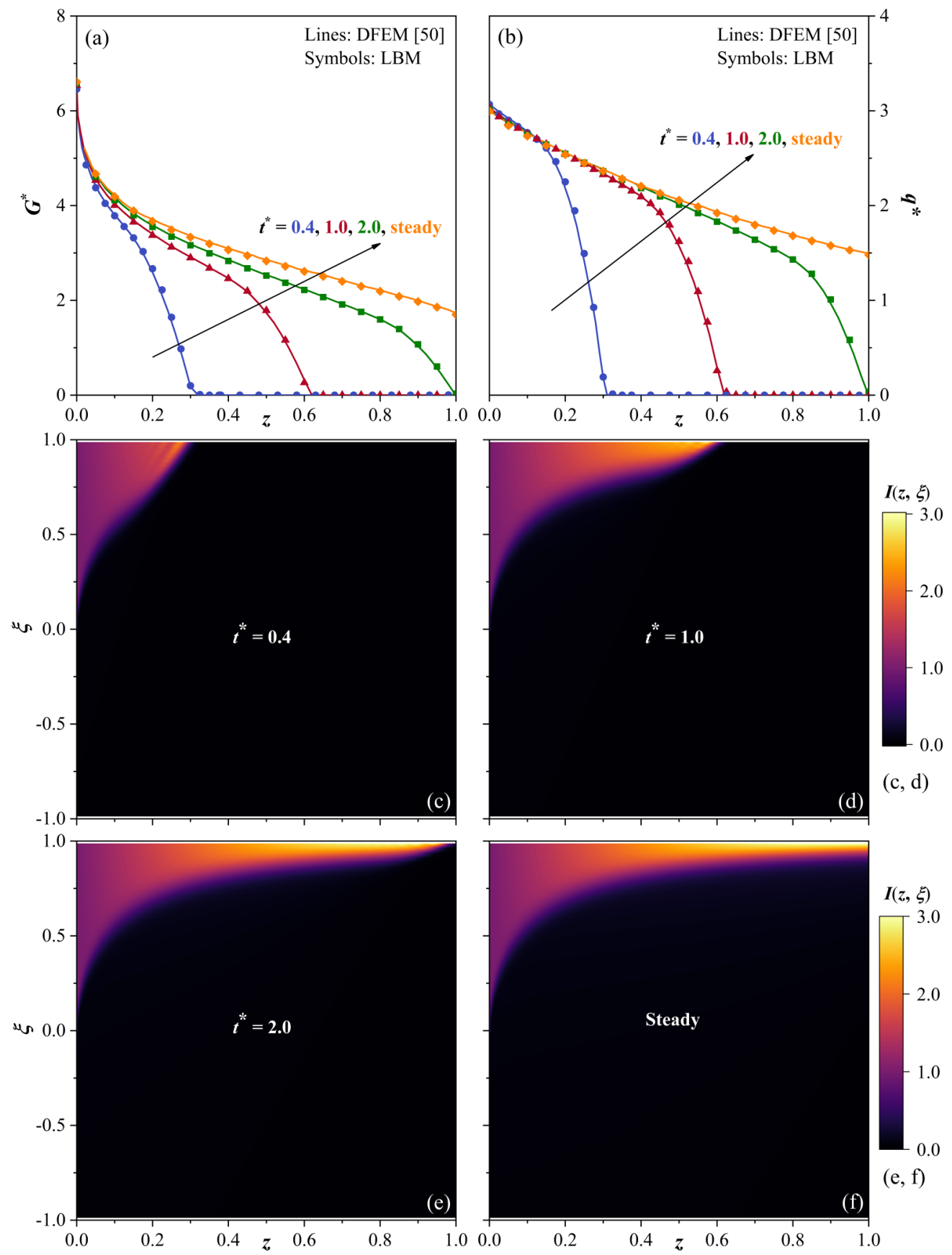

FIG. 3. Time evolutionary processes of incident radiation, radiative heat flux, and radiative intensity distribution in spatial and angular domains with $\tau_{L}=1.0, \omega=0.5$, and $n=1+2 z$. (a) Dimensionless incident radiation $G^{*}$. (b) Dimensionless radiative heat flux $q^{*}$. (c)-(f) Distribution of radiative intensity $I(z, \xi)$ at different times $t^{*}=0.4,1.0,2.0$, and steady state, respectively.

a universal model for RHT in media with arbitrary refractive index.

The first case we consider is the RHT in optically thin media with relatively small optical thickness. To investigate the performance of the present LBM in optically thick media with large optical thickness, the second case we consider is the radiative intensity solution in an infinite slab with a large optical thickness, $\tau_{L}=10$. The slab has two black surfaces. The temperatures of the upper and lower boundaries are $T_{L}=$ $1000 \mathrm{~K}$ and $T_{0}=0 \mathrm{~K}$, respectively. The temperature within the slab decreases from $T_{L}$ to $T_{0}$ linearly. The refractive index distribution is $n=1.2+0.6(z / L)$. Figures 5(a) and 5(b) show the dimensionless radiative intensity $\psi=I(\theta) / n_{L}^{2} \sigma T_{L}^{4}$ at the upper boundary of the slab with different scattering albedos, respectively, where $n_{L}$ is the refractive index of the upper boundary. The scattering albedo is $\omega=0$ (pure absorption) in Fig. 5(a), $\omega=0.5$ (isotropic scattering) in Fig. 5(b). The results of optical thickness $\tau_{L}=1.0$ are also presented in Fig. 5 for comparison. The results of the LBM and the improved integral equation method based on radiation distribution factors [53] exhibit excellent agreement, which demonstrates that the present LBM is accurate and stable for simulating RHT 

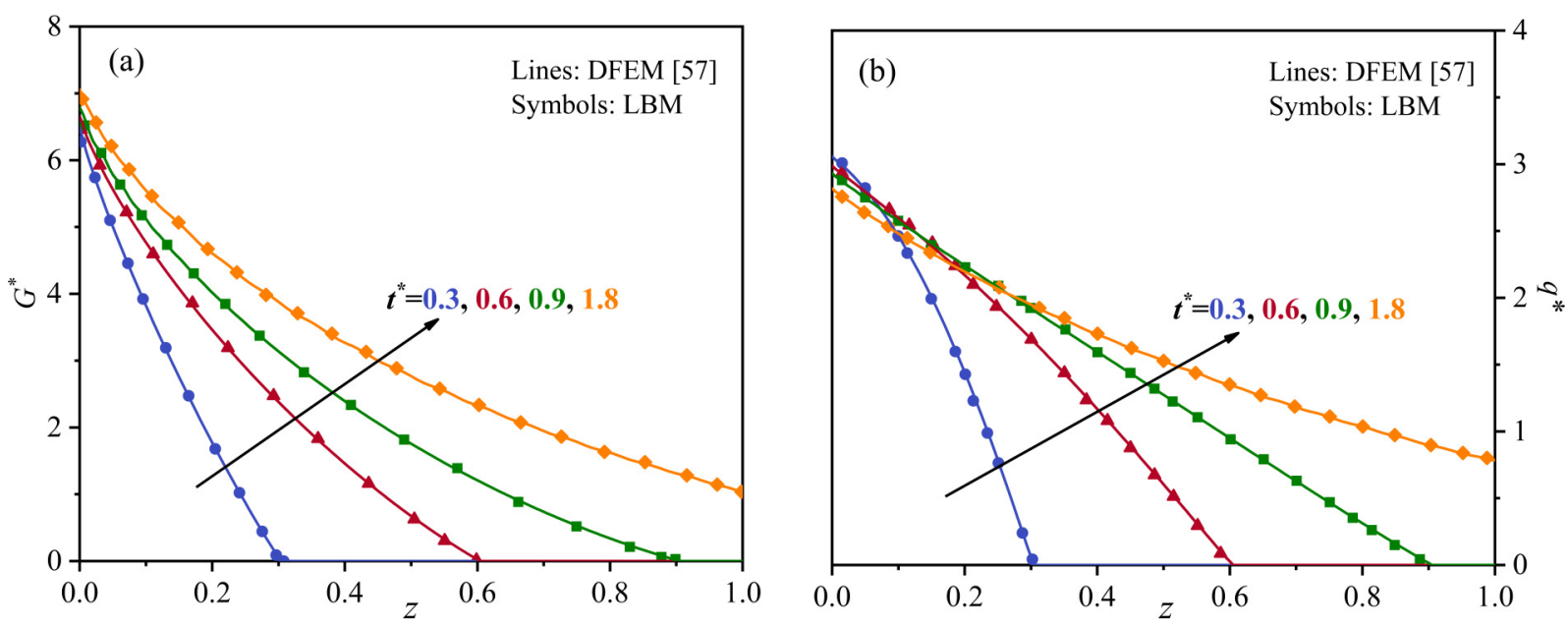

FIG. 4. Time evolutionary processes of incident radiation and radiative heat flux in the homogeneous slab with $\tau_{L}=1.0$, $\omega=0.5$, and constant refractive index $n=1$. (a) Dimensionless incident radiation $G^{*}$. (b) Dimensionless radiative heat flux $q^{*}$.

in graded-index media with different optical thicknesses. We also note that for the $\mathrm{D} n \mathrm{Q} m$ lattice structure and the LB model in this work, the convergence rate is embedded with respect to lattice size and holds for different optical thicknesses. The difference is that a greater relative error exists for optically thick media compared to optically thin media. Since a bigger extinction coefficient causes a sharper gradient in the radiation intensity distribution, then the bigger relative error is produced due to the sharper gradient.

The next case we consider is the steady-state RHT of an infinite slab in radiative equilibrium. The slab has two diffuse surfaces with emissivity $\varepsilon_{0}$ and $\varepsilon_{L}$, respectively. The temperatures of the two boundaries are $T_{0}=1000 \mathrm{~K}$ and $T_{L}=1500 \mathrm{~K}$, respectively. The refractive index distribution is $n=1.2+0.6(z / L)$. Figure 6 shows radiative equilibrium temperature distributions, in which the medium in Fig. 6(a) is nonscattering with $\varepsilon_{0}=\varepsilon_{L}=1$ and different optical thickness $\tau_{L}=0.01,1$, and 3, and the medium in Fig. 6(b) is linear-anisotropic scattering with scattering phase function
$\Phi=1+b \xi \xi^{\prime}, \omega=0.8, \tau_{L}=1$, and $\varepsilon_{0}=\varepsilon_{L}=1$ and different asymmetry factors $b=1,0$, and -1 . The LBM results with those of the curved ray-tracing (CRT) method [47] and the Chebyshev collocation spectral-discrete ordinates method [51] are presented in Fig. 6 for comparison. We can see that the LBM results are in good agreement with the benchmark results. The good agreement indicates that the present LBM is flexible and accurate for simulating steady-state RHT in graded-index media.

\section{B. Two-dimensional cases}

Through comparing the LBM results with other numerical results as well as theoretical results, we can conclude that the present LBM can efficiently and accurately capture one-dimensional transient and steady-state RHT processes in graded-index media. Also, the steady-state results can be naturally evolved from transient results. Finally, we will specifically test the performance of LBM on multidimensional
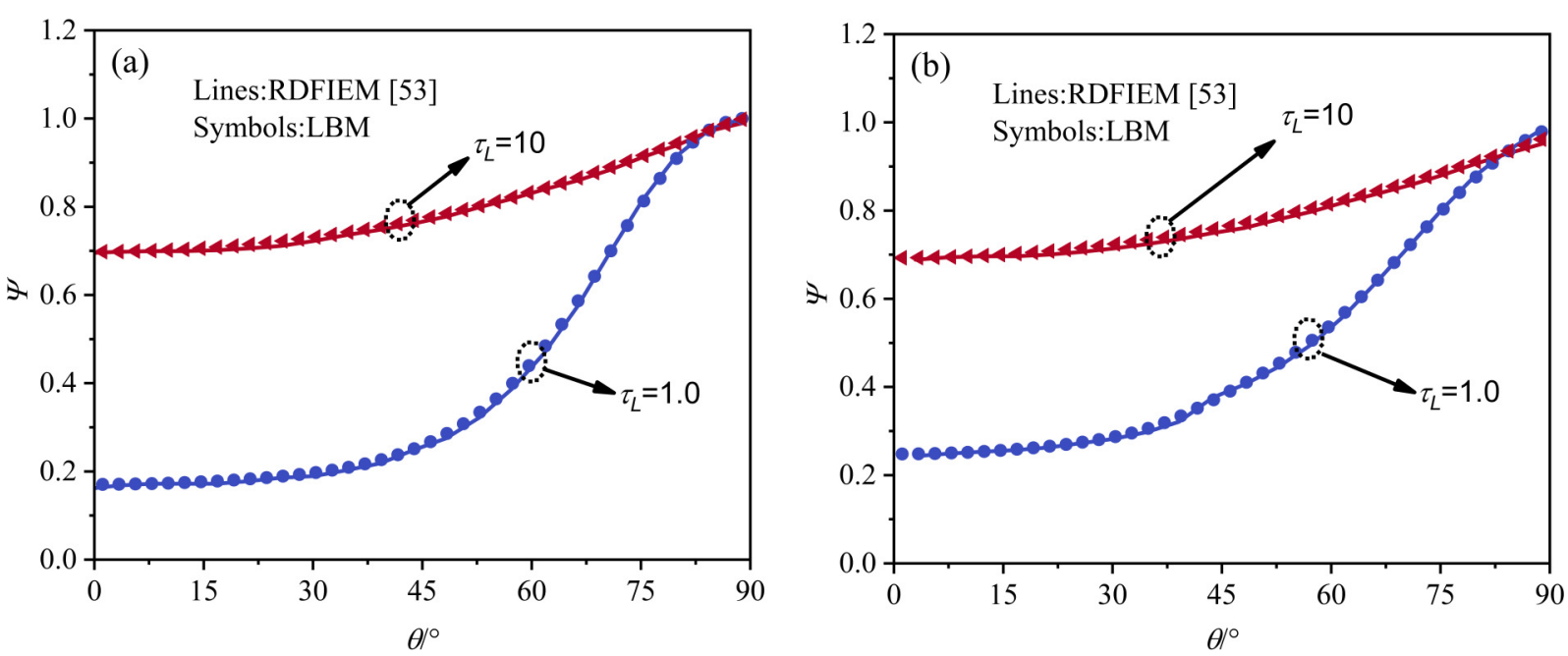

FIG. 5. The dimensionless radiative intensity at the upper boundary of the slab with different scattering albedos: (a) in the nonscattering medium and (b) in the isotropic scattering medium with $\omega=0.5$. 

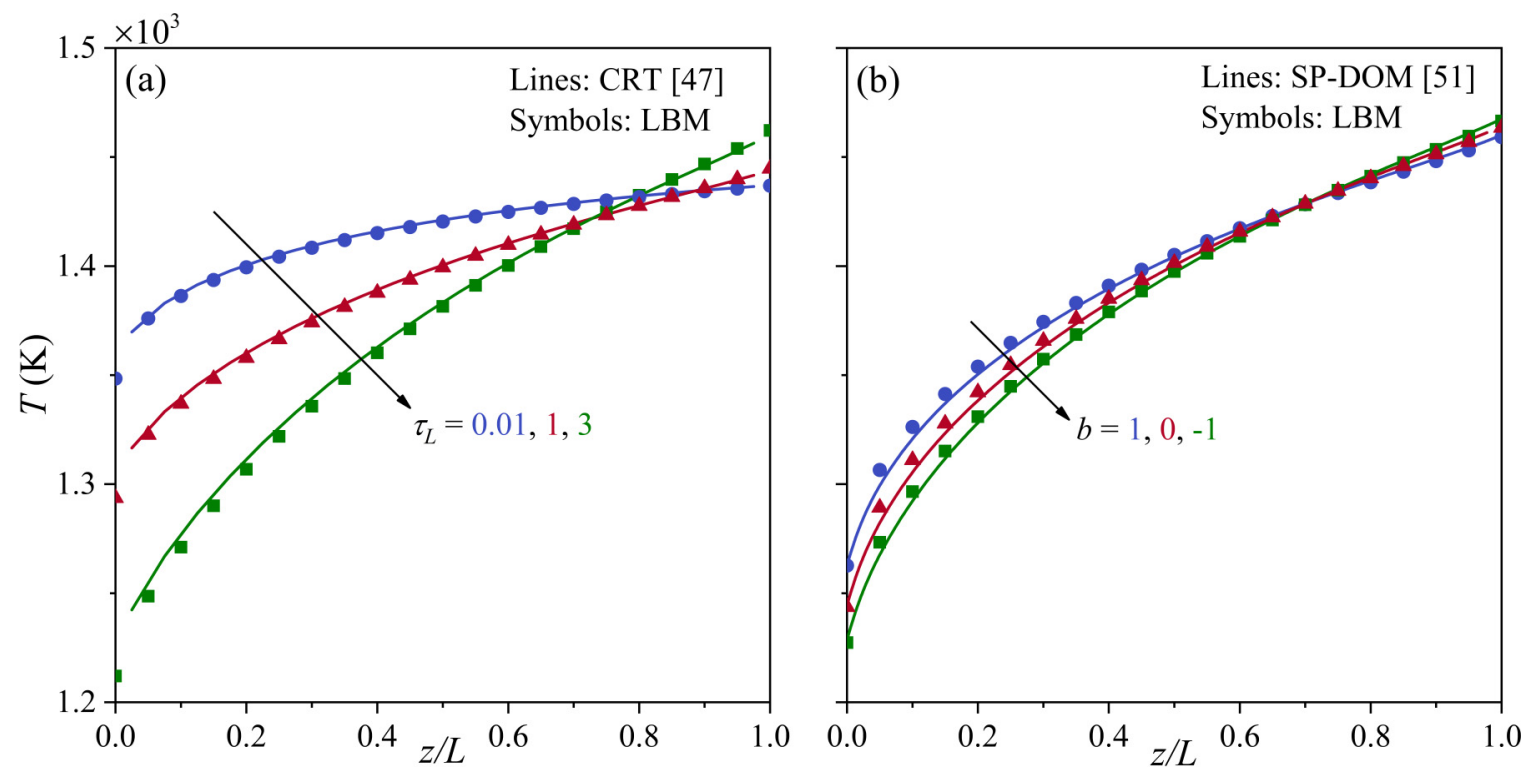

FIG. 6. Radiative equilibrium temperature distributions: (a) in the nonscattering medium with $\varepsilon_{0}=\varepsilon_{L}=1$ and (b) in the linear-anisotropic scattering medium with $\tau_{L}=1.0, \omega=0.8$, and $\varepsilon_{0}=\varepsilon_{L}=1$.

problems. Here, two-dimensional RHT in square enclosure with graded-index medium and opaque boundaries is studied. The lower, upper, left, and right walls of the square enclosure keep temperature $T_{w 1}, T_{w 2}, T_{w 3}$, and $T_{w 4}$, respectively. The medium in the square enclosure keeps temperature $T_{g}$, and the absorption coefficient $k_{a}$ and the scattering coefficient $k_{s}$ are uniform. Firstly, the square enclosure is set as black walls, optical thickness of $\tau_{L}=2$, and scattering albedo of $\omega=0.5$, with anisotropic scattering phase function $\Phi=1+\Omega \Omega^{\prime}$. The refractive index distribution is taken as $n=5 \sqrt{1-0.4356\left(x^{2}+y^{2}\right) / L^{2}}$. Two kinds of temperature distributions are considered: (1) $T_{w 1}=T_{w 2}=T_{w 3}=T_{w 4}=$ $0 \mathrm{~K}$ and $T_{g}=T_{\text {ref }}$, and (2) $T_{w 1}=T_{w 2}=T_{w 4}=T_{g}=0 \mathrm{~K}$ and $T_{w 3}=T_{\text {ref }}$. Figure 7(a) shows the dimensionless radiative heat flux $q_{w 1}^{*}=q_{w 1} / \sigma T_{\text {ref }}^{4}$ along the lower boundary with two temperature distributions, where $\sigma$ is the Stefan-Boltzmann constant. The results obtained by the present LBM and the Monte Carlo curved ray-tracing (MCCRT) method [49] are depicted in Fig. 7(a) for comparison. It is seen that the LBM results agree well with the MCCRT results. Next, the square enclosure is set as optical thickness of $\tau_{L}=0.1$, the scattering albedo of $\omega=1.0$ (pure isotropic scattering), and gray walls. The refractive index distribution is taken as $n=1+2(x+y) / L$. The lower wall keeps hot $\left(T_{w 1}=\right.$ $\left.T_{\text {ref }}\right)$, and the other walls and the media keep cold $\left(T_{w 2}=\right.$ $\left.T_{w 3}=T_{w 4}=T_{g}=0 \mathrm{~K}\right)$. The dimensionless radiative heat flux along the lower boundary with different emissivity $\varepsilon_{w}=$ $0.1,0.5$, and 1.0 are shown in Fig. 7(b). We can observe that the results obtained by the LBM and the finite-element method [48] exhibit good agreement. The good agreement between
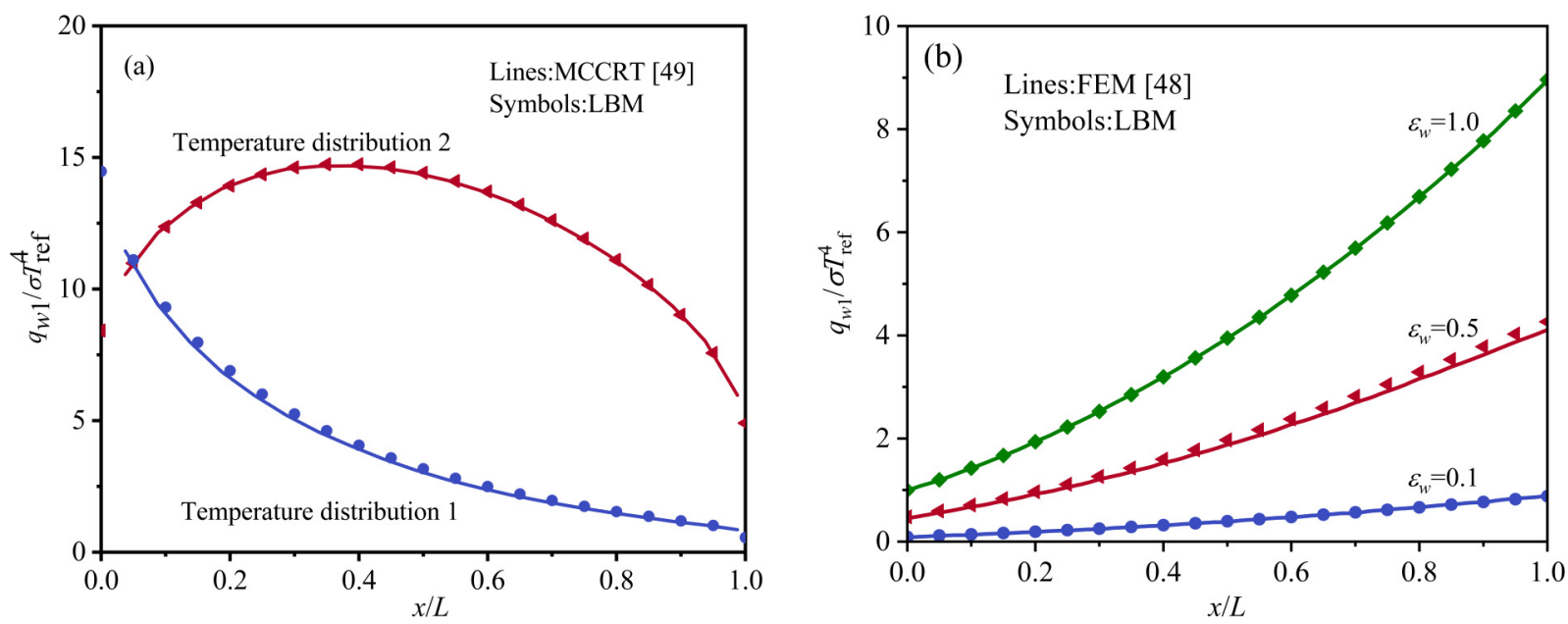

FIG. 7. Dimensionless radiative heat flux along the lower boundary: (a) square enclosure with black wall, anisotropic scattering medium, and two kinds of temperature distributions and (b) square enclosure with different wall emissivity $\varepsilon_{w}=0.1,0.5$, and 1.0 and pure isotropic scattering medium. 
the LBM results and other numerical results indicates that the present LBM can handle two-dimensional RHT in gradedindex media with various configurations accurately and stably.

Overall, the present LBM has been demonstrated to be capable of handling both transient and steady-state RHT in graded-index media. Compared with other traditional methods for RHT in graded-index media, the most striking feature of the present LBM is its mesoscopic property. Light travels along curved paths in graded-index media. Via the introduction of local radiative transfer direction and equilibrium distribution function, the present LBM enables a simple global solution of RHT in graded-index media by conducting collision and streaming processes in the LBE. This simple mesoscopic property avoids not only the extensive curved raytracing work of statistical methods but also complex matrix operations of methods for solving discrete partial differential equations.

\section{CONCLUSION}

In conclusion, we have established a mesoscopic LBM for RHT in graded-index media. The radiative intensity is simply obtained through the LBE conducting collision and streaming processes. Due to the introduction of the local radiative transfer direction, we further construct the equilibrium distribution function, source term, and auxiliary source term distribution function. Numerical results demonstrate that the present LBM is efficient, flexible, and accurate for simulating one-dimensional and two-dimensional transient and steadystate RHT in graded-index media. The mesoscopic property makes the present LBM promising to provide clear insights into RHT in complex media and pave the way for establishing a unified framework of LBM for convection, conduction, and thermal radiation heat transfer.

In the future work, we will work on extending the present LBM to more complex and challenging threedimensional RHT in graded-index media by adopting the three-dimensional local radiative transfer direction and lattice models.

\section{ACKNOWLEDGMENTS}

This work was financially supported by National Natural Science Foundation of China (Grants No. 51876004 and No. 52176051), Fundamental Research Funds for Central Universities (Grant No. YWF-21-BJ-J-818), and Academic Excellence Foundation of BUAA for Ph.D. Students.
[1] G. R. McNamara and G. Zanetti, Use of the Boltzmann Equation to Simulate Lattice-Gas Automata, Phys. Rev. Lett. 61, 2332 (1988).

[2] F. J. Higuera and J. Jiménez, Boltzmann approach to lattice gas simulations, Europhys. Lett. 9, 663 (1989).

[3] S. Chen and G. D. Doolen, Lattice Boltzmann method for fluid flows, Annu. Rev. Fluid Mech. 30, 329 (1998).

[4] C. K. Aidun and J. R. Clausen, Lattice-Boltzmann method for complex flows, Annu. Rev. Fluid Mech. 42, 439 (2010).

[5] P. Lallemand, L. S. Luo, M. Krafczyk, and W. A. Yong, The lattice Boltzmann method for nearly incompressible flows, J. Comput. Phys. 431, 109713 (2021).

[6] S. Chen, H. Chen, D. Martinez, and W. Matthaeus, Lattice Boltzmann Model for Simulation of Magnetohydrodynamics, Phys. Rev. Lett. 67, 3776 (1991).

[7] H. Chen, S. Kandasamy, S. Orszag, R. Shock, S. Succi, and V. Yakhot, Extended Boltzmann kinetic equation for turbulent flows, Science 301, 633 (2003).

[8] A. Mazloomi M, S. S. Chikatamarla, and I. V. Karlin, Entropic Lattice Boltzmann Method for Multiphase Flows, Phys. Rev. Lett. 114, 174502 (2015).

[9] X. Shan, Lattice Boltzmann in micro-and nano-flow simulations, IMA J. Appl. Math. 76, 650 (2011).

[10] X. He, S. Chen, and G. D. Doolen, A novel thermal model for the lattice Boltzmann method in incompressible limit, J. Comput. Phys. 146, 282 (1998).

[11] I. V. Karlin, D. Sichau, and S. S. Chikatamarla, Consistent two-population lattice Boltzmann model for thermal flows, Phys. Rev. E 88, 063310 (2013).

[12] M. Mendoza, B. M. Boghosian, H. J. Herrmann, and S. Succi, Fast Lattice Boltzmann Solver for Relativistic Hydrodynamics, Phys. Rev. Lett. 105, 014502 (2010).
[13] J. J. Wylie, D. L. Koch, and A. J. C. Ladd, Rheology of suspensions with high particle inertia and moderate fluid inertia, J. Fluid Mech. 480, 95 (2003).

[14] Z. G. Feng and E. E. Michaelides, The immersed boundarylattice Boltzmann method for solving fluid-particles interaction problems, J. Comput. Phys. 195, 602 (2004).

[15] M. Bernaschi, S. Melchionna, and S. Succi, Mesoscopic simulations at the physics-chemistry-biology interface, Rev. Mod. Phys. 91, 025004 (2019).

[16] Y. Shi, L. Wu, and X. Shan, Accuracy of high-order lattice Boltzmann method for non-equilibrium gas flow, J. Fluid Mech. 907, A25 (2020).

[17] N. Sawant, B. Dorschner, and I. V. Karlin, Consistent lattice Boltzmann model for multicomponent mixtures, J. Fluid Mech. 909, A1 (2020).

[18] W. S. Jiaung, J. R. Ho, and C. P. Kuo, Lattice Boltzmann method for the heat conduction problem with phase change, Numer. Heat Transfer, Part B 39, 167 (2001).

[19] J.-R. Ho, C.-P. Kuo, W.-S. Jiaung, and C.-J. Twu, Lattice Boltzmann scheme for hyperbolic heat conduction equation, Numer. Heat Transfer, Part B 41, 591 (2002).

[20] M. Eshraghi and S. D. Felicelli, An implicit lattice Boltzmann model for heat conduction with phase change, Int. J. Heat Mass Transfer 55, 2420 (2012).

[21] A. Nabovati, D. P. Sellan, and C. H. Amon, On the lattice Boltzmann method for phonon transport, J. Comput. Phys. 230, 5864 (2011).

[22] Y. Guo and M. Wang, Lattice Boltzmann modeling of phonon transport, J. Comput. Phys. 315, 1 (2016).

[23] Y. Guo and M. Wang, Lattice Boltzmann scheme for hydrodynamic equation of phonon transport, Int. J. Therm. Sci. 171, 107178 (2022). 
[24] B. Shi and Z. Guo, Lattice Boltzmann model for nonlinear convection-diffusion equations, Phys. Rev. E 79, 016701 (2009).

[25] R. Blaak and P. M. A. Sloot, Lattice dependence of reactiondiffusion in lattice Boltzmann modeling, Comput. Phys. Commun. 129, 256 (2000).

[26] Y. Guangwu, A lattice Boltzmann equation for waves, J. Comput. Phys. 161, 61 (2000).

[27] S. Palpacelli and S. Succi, The quantum lattice Boltzmann equation: Recent developments, Commun. Comput. Phys. 4, 980 (2008)

[28] P. Asinari, S. C. Mishra, and R. Borchiellini, A lattice Boltzmann formulation for the analysis of radiative heat transfer problems in a participating medium, Numer. Heat Transfer, Part B 57, 126 (2010).

[29] Y. Ma, S. K. Dong, and H. Tan, Lattice Boltzmann method for one-dimensional radiation transfer, Phys. Rev. E 84, 016704 (2011).

[30] H. Bindra and D. V. Patil, Radiative or neutron transport modeling using a lattice Boltzmann equation framework, Phys. Rev. E 86, 016706 (2012).

[31] Y. Zhang, H. Yi, and H. Tan, One-dimensional transient radiative transfer by lattice Boltzmann method, Opt. Express 21, 24532 (2013).

[32] A. Mink, G. Thäter, H. Nirschl, and M. J. Krause, A 3D lattice Boltzmann method for light simulation in participating media, J. Comput. Sci. 17, 431 (2016).

[33] H. L. Yi, F. J. Yao, and H. P. Tan, Lattice Boltzmann model for a steady radiative transfer equation, Phys. Rev. E 94, 023312 (2016).

[34] Y. Wang, L. Yan, and Y. Ma, Lattice Boltzmann solution of the transient Boltzmann transport equation in radiative and neutron transport, Phys. Rev. E 95, 063313 (2017).

[35] X. Liu, Y. Huang, C. H. Wang, and K. Zhu, Solving steady and transient radiative transfer problems with strong inhomogeneity via a lattice Boltzmann method, Int. J. Heat Mass Transfer 155, 119714 (2020).

[36] X. Liu, Y. Huang, C.-H. Wang, and K. Zhu, A multiplerelaxation-time lattice Boltzmann model for radiative transfer equation, J. Comput. Phys. 429, 110007 (2021).

[37] X. Liu, K. Zhu, J. Shao, and Y. Huang, A lattice Boltzmann scheme for polarized radiative transfer in planetary atmospheres, Astron. J. 162, 122 (2021).

[38] X. Liu, H. Wu, K. Zhu, and Y. Huang, Lattice Boltzmann model for multidimensional polarized radiative transfer: Theory and application, Optica 8, 1136 (2021).

[39] E. W. Marchand, Gradient Index Optics (Academic Press, New York, 1978).

[40] M. Born and E. Wolf, Principles of Optics (Cambridge University Press, Cambridge, UK, 1999).

[41] X. Xiao, C. W. Choi, and I. K. Puri, Temperature measurements in steady two-dimensional partially premixed flames using laser interferometric holography, Combust. Flame 120, 318 (2000).
[42] P. M. Kowalski and D. Saumon, Radiative transfer in the refractive atmospheres of very cool white dwarfs, Astrophys. J. 607, 970 (2004)

[43] Y. F. Tsay, B. Bendow, and S. S. Mitra, Theory of the temperature derivative of the refractive index in transparent crystals, Phys. Rev. B 8, 2688 (1973).

[44] P. Ben Abdallah and V. Le Dez, Temperature field inside an absorbing-emitting semi-transparent slab at radiative equilibrium with variable spatial refractive index, J. Quant. Spectrosc. Radiat. Transfer 65, 595 (2000).

[45] P. Ben Abdallah and V. Le Dez, Thermal emission of a two-dimensional rectangular cavity with spatial affine refractive index, J. Quant. Spectrosc. Radiat. Transfer 66, 555 (2000).

[46] D. Lemonnier and V. Le Dez, Discrete ordinates solution of radiative transfer across a slab with variable refractive index, J. Quant. Spectrosc. Radiat. Transfer 73, 195 (2002).

[47] Y. Huang, X. L. Xia, and H. P. Tan, Temperature field of radiative equilibrium in a semitransparent slab with a linear refractive index and gray walls, J. Quant. Spectrosc. Radiat. Transfer 74, 249 (2002).

[48] L. H. Liu, L. Zhang, and H. P. Tan, Finite element method for radiation heat transfer in multi-dimensional graded index medium, J. Quant. Spectrosc. Radiat. Transfer 97, 436 (2006).

[49] L. H. Liu, Benchmark numerical solutions for radiative heat transfer in two-dimensional medium with graded index distribution, J. Quant. Spectrosc. Radiat. Transfer 102, 293 (2006).

[50] L. H. Liu and P. F. Hsu, Analysis of transient radiative transfer in semitransparent graded index medium, J. Quant. Spectrosc. Radiat. Transfer 105, 357 (2007).

[51] Y.-S. Sun and B.-W. Li, Chebyshev collocation spectral method for one-dimensional radiative heat transfer in graded index media, Int. J. Therm. Sci. 48, 691 (2009).

[52] H. L. Yi, C. H. Wang, and H. P. Tan, Transient radiative transfer in a complex refracting medium by a modified Monte Carlo simulation, Int. J. Heat Mass Transfer 79, 437 (2014).

[53] L. Y. Wei, H. Qi, Y. Li, S. Wen, Y. T. Ren, M. A. Islam, and L. M. Ruan, Improved integral equation method based on radiation distribution factors for high-resolution radiative intensity in graded index media, Int. J. Therm. Sci. 138, 393 (2019).

[54] Y. Zhang, H. L. Yi, and H. P. Tan, The lattice Boltzmann method for one-dimensional transient radiative transfer in graded index gray medium, J. Quant. Spectrosc. Radiat. Transfer 137, 1 (2014).

[55] L. Rezzolla and O. Zanotti, Relativistic Hydrodynamics (Oxford University Press, Oxford, 2013).

[56] Z. L. Guo, C. G. Zheng, and B. C. Shi, Non-equilibrium extrapolation method for velocity and pressure boundary conditions in the lattice Boltzmann method, Chin. Phys. Soc. 11, 366 (2002).

[57] L. H. Liu and L. J. Liu, Discontinuous finite element approach for transient radiative transfer equation, J. Heat Transfer 129 , 1069 (2007). 